\title{
An Analysis of Improving Memory Performance \\ Based on EEG Alpha and Theta Oscillations
}

\author{
Tianbao Zhuang \& Hong Zhao \\ Graduate School of Innovative Life Science \\ University of Toyama \\ Toyama, Japan \\ Tel: 81-090-3886-9168Ｅ-mail: bfztb@sina.com \\ Zheng Tang \\ Faculty of Engineering \\ University of Toyama \\ Toyama, Japan \\ E-mail: ztang@eng.u-toyama.ac.jp
}

\begin{abstract}
Evidence is presented that EEG oscillations in the alpha and theta band reflect memory performance in particular. Good performance is related to two types of EEG phenomena: a tonic increase in alpha but a decrease in theta power, and a large phasic decrease in alpha but increase in theta, depending on the type of memory demands. In a similar way as brain volume does, upper alpha power increases from early childhood to adulthood, whereas the opposite holds true for the late part of the lifespan. Alpha power is lowered and theta power enhanced in subjects with a variety of different neurological disorders. Furthermore, after sustained wakefulness and during the transition from waking to sleeping when the ability to respond to external stimuli ceases, upper alpha power decreases, whereas theta increases. Event-related changes indicate that the extent of upper alpha desynchronization is positively correlated with (semantic) long-term memory performance, whereas theta synchronization is positively correlated with the ability to encode new information.
\end{abstract}

Keywords: EEG, Alpha, Theta, Oscillation, Memory

\section{Introduction}

Scalp EEG signals are produced by partial synchronization of neuronal-scale field potentials across areas of cortex of centimetre-squared scale. Although once viewed by some as a form of brain 'noise', it appears increasingly probable that this synchronization optimizes relations between spike-mediated 'top-down' and 'bottom-up' communication, both within and between brain areas. This optimization might have particular importance during motivated anticipation of, and attention to, meaningful events and associations - and in response to their anticipated consequences (A. von Stein et al. 2000.)( P. Fries et al. 2001.)( E. Salinas and T.J. Sejnowski. 2001.). In a physiological sense, EEG power reflects the number of neurons that discharge synchronously.During the few years, alpha and theta oscillations have attracted considerable interest. It is the purpose of the present article to show that EEG power is indeed related to memory performance, but in a complex and partly non-linear way. Within the alpha frequency range EEG power is positively related to cognitive performance and brain maturity, whereas the opposite holds true for the theta frequency range. Alpha and theta reactivity as well as event-related changes in alpha and theta band power show yet another pattern of results. During actual task demands the extent of alpha power suppression is positively correlated with memory performance in particular, whereas again the opposite holds true for the theta band. The extent of theta synchronization is related to good performance.

\section{Design and method}

\subsection{Subjects}

A sample of 10 right handed students ( 5 males and 5 females) participated in the experiment. Their mean age was 23.5 years. Before participating in the experiment, subjects were asked about the hand they use in different tasks such as 
handwriting, throwing a ball, etc. A subject was considered right-handed if he/she indicated to use the right hand for all of these different tasks.

\subsection{Design}

Subjects had to respond by pressing a 'yes' response key if the letter or number of the frame was contained in the memory set, otherwise with 'no'. Each subject was tested under all of the experimental conditions. Presentation sequence was counterbalanced between subjects.

\subsection{Apparatus}

EEG-signals were amplified by a 32-channel biosemi system (frequency response: 0.15 to $30 \mathrm{~Hz}$ ), subjected to an anti-aliasing filterbank (cut-off frequency: $30 \mathrm{~Hz}, 110 \mathrm{~dB} / \mathrm{octave}$ ) and were then converted to a digital format via a 32-channel A/D converter. Sampling rate was $256 \mathrm{~Hz}$. During data acquisition, EEG signals were displayed online on a high resolution monitor and stored on disk.

\subsection{Recordings}

A set of 25 silver electrodes, attached with a glue paste to the scalp was used to record EEG-signals. Thirteen electrodes were placed according to the International Electrode (10-20) Placement-System, at F3, F4, Fz, C3, C4, Cz, T3, T4, P3, $\mathrm{P} 4, \mathrm{Pz}, \mathrm{O} 1$ and $\mathrm{O} 2$. From the remaining twelve electrodes, 4 electrodes were placed over parieto-occipital areas (PO3, PO1, PO2, PO4), 4 electrodes were placed over centro-parietal areas (CP5, CP1, CP2, CP6), and 4 electrodes were placed over fronto-central areas (FC5, FC1, FC2, FC6). ( Figure 1) In addition to the 25 electrodes described above, two ear lobe electrodes (termed A1 and A2), were attached to the left and right ear. The EOG was recorded from 2 pairs of leads in order to register horizontal and vertical eye movements.

\section{Conclusion}

\subsection{Event-related changes in the alpha and theta band}

Since the work of Berger it was suggested that visual (or other sensory) task demands and visual attention in particular are the primary factors that lead to a suppression of the alpha rhythm (W.J. Ray and H.W. Cole. 1985.). In using event-related desynchronization (ERD), a method introduced originally by Pfurtscheller and Aranibar (G. Pfurtscheller and A. Aranibar. 1977.), recent research has revealed a much more complex picture.

A typical example of an EEG epoch which is used for measuring event-related desynchronization (ERD) is shown in( Figure 2). During the first second of the epoch, which is called reference interval, the subject shows pronounced rhythmic alpha activity. Subjects run through many trials and, thus, the anticipation of the warning signal already causes alpha to desynchronize even before the warning signal actually appears. As spectral analysis shows, this attentional effect is reflected only in the lower alpha band. The subjects' task was to read a visually presented word and to make a semantic judgment by responding 'yes' to a word denoting a living and 'no' to a word denoting a non-living object. Before a word appeared a warning signal is presented. Subjects had to judge a total of 96 words. The basic principle for measuring ERD is that alpha shows a typical phasic change over the time course of a trial. After a response, the subject relaxes and awaits the presentation of the next stimulus. This state of relaxed but alert wakefulness is reflected by a pronounced alpha activity during the reference interval which precedes each trial. Even before the warning signal actually appears, the alpha rhythm becomes suppressed, because the subject anticipates the beginning of the next trial.

The measurement of ERD is done in several steps. First, the EEG is band pass filtered within defined frequency bands, the filtered data are squared and then averaged within consecutive time intervals (of e.g., $125 \mathrm{~ms}$ ). Second, the obtained data are averaged over the number of epochs. Third, band power changes are expressed as the percentage of a decrease or increase in band power during a test as compared to a reference interval by using the following simple formula: $\mathrm{ERD}=(($ band power reference-band power test $) /($ band power reference $)) \times 100$. Note that desynchronization is reflected by positive ERD values, whereas event-related synchronization (ERS) is reflected by negative ERD values (G. Pfurtscheller, A. Stancak, Jr. and C. Neuper. 1996.).

The appropriate selection of frequency bands is one of the most critical issues when using ERD or related measures such as changes in induced band power. Due to large interindividual differences of alpha frequency, large portions of alpha power may fall outside a fixed frequency window and invite misleading interpretations. In order to avoid these and related problems that arise with fixed frequency windows, we suggested to use alpha frequency $f(i)$, averaged over all leads, as an anchor point to adjust frequency bands individually for each subject. Then, four frequency bands with a width of $2 \mathrm{~Hz}$ can be defined in relation to $f(i)$ that cover the traditional theta and alpha frequency range from about 4-12 Hz (depending on the actual $f(i)$ for each subject). The frequency bands obtained by this method are termed: theta $(f(i)-6$ to $f(i)-4)$; lower 1 alpha $(f(i)-4$ to $f(i)-2)$; lower 2 alpha $(f(i)-2$ to $f(i))$ and upper alpha $(f(i)$ to $f(i)+2)$. For each subject, ERD is calculated within these individually determined frequency bands. We have shown in several studies that fixed frequency bands blur the specific relationships between cognitive performance and ERD, we otherwise are able to observe (as an example, see Figure 3).where subjects did not know in advance that a recall test will be carried out later. 
Data represent the time period of $1000 \mathrm{~ms}$ poststimulus after presentation onset of a word during encoding and are averaged over all recording sites. (A) Those words that can be remembered in a later recall task show a significantly larger task related increase in theta power (negative ERD values) during encoding as compared to words that cannot be remembered later. The respective differences in the three alpha bands are not significant. (B) In the theta band not only (negative) ERD (reflecting the percentage of an increase in power with respect to a reference interval) but also absolute power too is significantly larger for remembered words but only if frequency bands are adjusted individually (as for the ERD analysis) to IAF. (C) If instead fixed frequency windows are used, no significant differences can be observed.3.2 3.2 The relationship between desynchronization, synchronization and absolute power

As the results by Klimesch et al. (W. Klimesch, M. Doppelmayr, H. Russegger and T. Pachinger. 1996.)( W. Klimesch, M. Doppelmayr, T. Pachinger and H. Russegger. 1997.) have shown, the extent of theta synchronization and upper alpha desynchronization are related to episodic and semantic memory performance, respectively. Although these findings reveal very specific effects, they are in good agreement with the well known fact that the amount of alpha desynchronization generally is related to the relevance and/or difficulty of a task. The more demanding or relevant a task, the stronger the amount of alpha suppression or ERD (W. Klimesch, G. Pfurtscheller, W. Mohl and H. Schimke. 1990.). In a pure logical sense one would expect that the amount of desynchronization should depend on absolute power. Only if there is sufficient activity during a reference or resting interval would there be a possibility of a large extent of power suppression during task performance. A similar relationship may also be expected on the basis of physiological considerations. It would be quite plausible to assume that after a difficult task, a rebound of alpha activity takes place that lasts even into the reference interval of the next following trial (epoch). If this occurs trial after trial, the percentage of desynchronization (ERD) would clearly be linked to the power of the reference interval.

For all of the three alpha bands, the results clearly indicate that large band power in the reference interval is associated with a large amount of desynchronization (alpha suppression) during task performance. Most interestingly, the opposite holds true for the theta band. Here, small reference power is related to a large amount of synchronization or increase in power. Thus, the extent of alpha desynchronization and theta synchronization depend on the magnitude of absolute band power, but in opposite ways. With respect to the broad alpha band (7-14 Hz), similar findings were reported by Salenius et al. (S. Salenius, M. Kajola, W.L. Thompson, S. Kosslyn and R. Hari. 1995.). The interesting conclusion, thus, is that the reactivity in band power which reflects task performance can be predicted from the amount of absolute power as measured during a resting state.

With respect to the relationship between memory performance and absolute power, the reported findings allow us to make an important prediction. We would expect that good as compared to bad memory performers show significantly more power in the upper alpha but less power in the theta band. This result would be expected even when the EEG is measured during a resting phase. This hypothesis was clearly supported by two recent studies (F. Vogt, W. Klimesch and M. Doppelmayr. 1997.)( W. Klimesch, F. Vogt, M. 1998.), as the example shown in Figure 4 indicates. The ongoing EEG was analysed for 4 min and arbitrarily segmented in epochs of $4 \mathrm{~s}$ in order to achieve a frequency resolution of $0.25 \mathrm{~Hz}$. Significant results (of $t$-tests between good and bad memory performers) are marked by one asterisk (for the 5\%-level) or two vertically arranged asterisks (for the $1 \%$-level). Good memory performers show significantly more upper alpha but less theta power. Similar results were found for all recording sites and even during resting sessions with eyes open and closed. Similar findings were reported by Jausovec (N. Jausovec. 1996.). He found that highly intelligent subjects have significantly more absolute power in the broad alpha frequency range $(7.5-13 \mathrm{~Hz})$ than less intelligent subjects.

\subsection{General conclusions and physiological considerations}

The most important conclusion is that the amount of EEG power in the theta and alpha frequency range is indeed related to memory performance in particular, if a double dissociation between absolute and event-related changes in alpha and theta power is taken into account. This double dissociation is characterized by the fact that during a resting state

(i) small theta power but large alpha power (particularly in the frequency range of the upper alpha band) indicates good performance, whereas the opposite holds true for event-related changes, where

(ii) a large increase in theta power (synchronization) but a large decrease in alpha power (desynchronization) reflect good memory performance in particular.

A comparison with findings about the hippocampal theta rhythm in animals reveals that in response to increasing encoding demands, hippocampal theta synchronizes in a small frequency window, just as the human theta (scalp) EEG does (for an extensive review of this issue see Ref. (W. Klimesch. 1996.)). Theta synchronization is due to an increase in the duration of multi-unit population bursts and to an increase in rhythmicity of these bursts which have the same frequency as theta. Convincing evidence for the hypothesis that theta synchronization is related to the encoding of new information comes from the fact that LTP is closely linked to the synchronous activity of the hippocampal theta rhythm: 
- LTP can be best induced with stimulation patterns that mimic theta rhythm (J. Larson, D. Wong and G. Lynch. 1986.).

- LTP has been demonstrated in several brain regions, but it is most robust and, thus, has been studied most extensively in the hippocampus.

- The induction of LTP occurs primarily during the positive phase of the theta rhythm.

- The strength of the induced LTP increases linearly with increasing theta power.

- Pharmacological manipulations demonstrate that drugs which decrease theta activity also block learning, whereas drugs that promote the theta rhythm (and, thus, enhance the induction of LTP) also facilitate learning.

These findings support the view that hippocampal theta is important for the induction of LTP and is related to the encoding of new information in a similar way as LTP is. Consequently, we may assume that our results which suggest a close relationship between theta synchronization and the encoding of new information reflect theta activity that is induced into the cortex via cortico-hippocampal feedbackloops for a comprehensive review on this topic). Further evidence for this interpretation comes from an interesting study by Gevins et al. (A. Gevins, M.E. Smith, H. Leong, L. McEvoy, S. Whitfield, R. Du and G. Rush. 1998.), who used a new method to spatially sharpen the EEG with magnetic resonance imaging-based finite element deblurring. These authors found a frontal midline theta rhythm which increased with increasing memory load. Most interestingly, dipole models localized this signal to the region of the anterior cingulate cortex which is part of the Papez circuit and, thus, is linked with the hippocampal formation via complex feedback or 'reentrant' loops.

It should be noted that there are two different types of theta synchronization. One type of synchronization is related to an increase in power within a narrow frequency band in the range of peak theta frequency. The second form of theta synchronization refers to irregular slow activity (ISA) which also is termed large irregular activity (LIA). Irregular slow activity dominates during slow wave sleep (SWS) and shows increased power outside the theta peak range. This type of synchronization is not related to the increased power of a dominant rhythm within a narrow frequency band, but rather to an increase in power over a broad range. It may be explained in terms of irregular oscillatory epochs of the type Buzsaki et al. (G. Buzsaki, Z. Horvath, R. Urioste, J. Hetke and K. Wise. 1992.) have described. These irregular oscillatory epochs which occur over a comparatively broad frequency range are not coupled to the 'coordinating' force of the theta rhythm and are not related to the encoding of new information. It appears plausible to assume that the broad band increase in theta power reflects a state in which the ability to encode new information is reduced or even blocked whereas the narrow band synchronization during regular rhythmic theta activity reflects event-related theta synchronization that is closely linked to the encoding of new information (or 'recoding' during REM).

In contrast to theta, the physiological mechanisms underlying alpha desynchronization appear more complex and at the first glance even paradoxical. Whereas alpha synchronization occurs during alert wakefulness, desynchronization reflects actual cognitive information processes. Alpha synchronization is a state in which millions of cortical neurons oscillate synchronously with the same phase and within a comparatively narrow frequency band. Desynchronization seems to imply that different oscillators within the alpha band are no longer coupled and start to oscillate with different frequencies. These different oscillators most likely reflect the synchronous activity of more local cortical or thalomocortical networks and are, thus, termed 'local' or 'functional' alphas. For each of the local alphas all neurons may still show a regular pattern of synchronous oscillation.

This basic EEG-phenomenon of large scale alpha synchronization (during mental inactivity) and desynchronization (during mental activity) which probably reflects a complex pattern of micro scale synchronization provides us with a preliminary but nonetheless important understanding of how information may be processed in the brain: Large scale alpha synchronization blocks information processing because very large populations of neurons oscillate with the same phase and frequency. In contrast, alpha desynchronization reflects actual cognitive processes because different neuronal networks start to oscillate at different frequencies and with different phases.

Research focusing on gamma oscillations in the visual cortex have shown that synchronous oscillatory discharge patterns reflect an elementary visual encoding process. These results, obtained with microelectrodes, are a good example of a microscale synchronization. With respect to the human scalp EEG, it is a matter of resolution, whether or not we may speak of synchronization or desynchronization. Even if the EEG desynchronizes, a large number of different networks may still show synchronous oscillations on a microscale level. In order to discriminate between these two different types of synchronization, the synchronous activity of large cortical areas reflecting mental inactivity is termed type 1 synchronization, whereas the regular synchronous oscillatory discharge pattern of selected and comparatively small cortical areas is termed type 2 synchronization. Type 1 synchronization, reflecting the summed activity of a large number of cell assemblies is a strong signal that can easily be recorded by macroelectrodes from the scalp. In contrast, the synchronous discharge of a small number of cell assemblies is a rather weak signal for the human scalp EEG. Thus, the behavior of the alpha rhythm can be explained by type 1 synchronization reflecting mental inactivity and type 2 synchronization reflecting mental activity. The behavior of the theta rhythm, on the other hand, can 
be described by type 2 regular synchronization (reflecting mental activity) and by type 1 irregular synchronization (reflecting mental inactivity). The general conclusion, thus, is that regular type 2 synchronization is that oscillatory mode in all of the frequency bands that reflects actual information processing in the brain.

In an attempt to integrate results from memory research in divergent fields such as cognitive psychology, neuroanatomy and neurophysiology, Klimesch has suggested that type 2 synchronization, generated by thalamo-cortical and cortico-cortical feedback loops provides an ideal framework for describing spreading activation processes in semantic long-term memory. Thus, it may be assumed that type 2 synchronization as measured by (upper) alpha desynchronization reflects search and retrieval processes in semantic long-term memory which are induced into the cortex by thalomo-cortical feedback loops. For the theta band it is suggested that hippocampo-cortical pathways induce synchronous oscillations within a narrow frequency window of the theta peak into widely distributed assemblies in the cortex, thereby binding different parts of cell assemblies together. This binding process forms the basis for encoding new information. This interpretation also implies that cognitive performance is closely linked to type 2 synchronization in thalamo- and hippocampo-cortical networks.

\section{References}

A. Gevins, M.E. Smith, H. Leong, L. McEvoy, S. Whitfield, R. Du and G. Rush, Monitoring working memory load during computer-based tasks with EEG pattern recognition methods. Human Factors 40 (1998), pp. 79-91.

A. von Stein et al., Top-down processing mediated by interareal synchronization. Proc. Natl. Acad. Sci. U. S. A. 97 (2000), pp. 14748-14753.

E. Salinas and T.J. Sejnowski, Correlated neuronal activity and the flow of neural information. Nat. Rev. Neurosci. 2 (2001), pp. 539-550.

F. Vogt, W. Klimesch and M. Doppelmayr, High frequency components in the alpha band and memory performance. $J$. Clin. Neurophysiol. 15 (1997), pp. 167-172.

G. Buzsaki, Z. Horvath, R. Urioste, J. Hetke and K. Wise, High-frequency network oscillation in the hippocampus. Science 256 (1992), pp. 1025-1027.

G. Pfurtscheller and A. Aranibar, Event-related cortical desynchronization detected by power measurement of scalp EEG. Electroencephalogr. Clin. Neurophysiol. 42 (1977), pp. 817-826.

G. Pfurtscheller, A. Stancak, Jr. and C. Neuper, Event-related synchronization (ERS) in the alpha band-an electrophysiological correlate of cortical idling: a review. Int. J. Psychophysiol. 24 (1996), pp. 39-46.

J. Larson, D. Wong and G. Lynch, Patterned stimulation at the theta frequency is optimal for the induction of hippocampal long-term potentiation. Brain Res. 368 (1986), pp. 347-350.

N. Jausovec, Differences in EEG alpha activity related to giftedness. Intelligence 23 (1996), pp. 159-173.

P. Fries et al., Modulation of oscillatory neuronal synchronization by selective visual attention. Science 291 (2001), pp. $1560-1563$.

S. Salenius, M. Kajola, W.L. Thompson, S. Kosslyn and R. Hari, Reactivity of magnetic parieto-occipital alpha rhythm during visual imagery. Electroencephalogr. Clin. Neurophysiol. 95 (1995), pp. 453-462.

W. Klimesch, F. Vogt, M. Doppelmayr, Interindividual differences in alpha and theta power reflect memory performance, Intelligence, 1998 (in press).

W. Klimesch, G. Pfurtscheller, W. Mohl and H. Schimke, Event-related desynchronization, ERD-mapping and hemispheric differences for words and numbers. Int. J. Psychophysiol. 8 (1990), pp. 297-308.

W. Klimesch, M. Doppelmayr, H. Russegger and T. Pachinger, Theta band power in the human scalp EEG and the encoding of new information. NeuroReport 7 (1996), pp. 1235-1240.

W. Klimesch, M. Doppelmayr, T. Pachinger and H. Russegger, Event-related desynchronization in the alpha band and the processing of semantic information. Cogn. Brain Res. 6 (1997), pp. 83-94.

W. Klimesch, Memory processes, brain oscillations and EEG synchronization. Int. J. Psychophysiol. 24 (1996), pp. 61-100.

W.J. Ray and H.W. Cole, EEG alpha activity reflects attentional demands, and beta activity reflects emotional and cognitive processes. Science 228 (1985), pp. 750-752. 


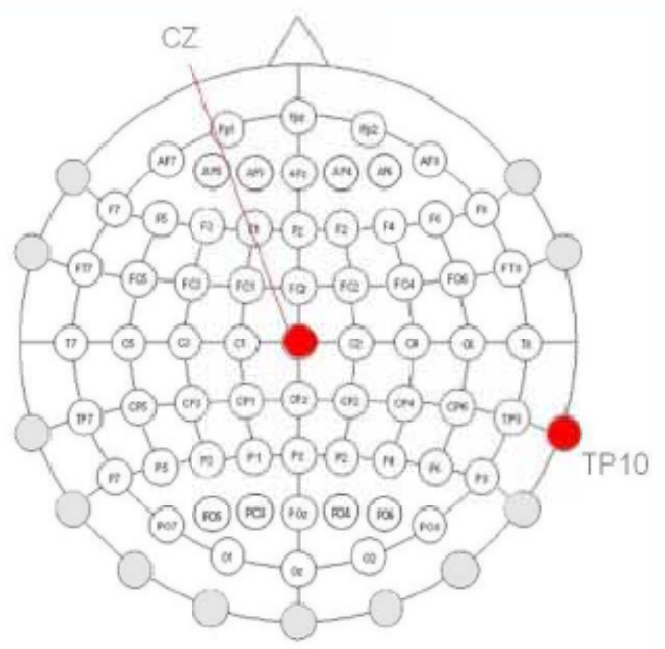

Figure 1. International Electrode Placement- System

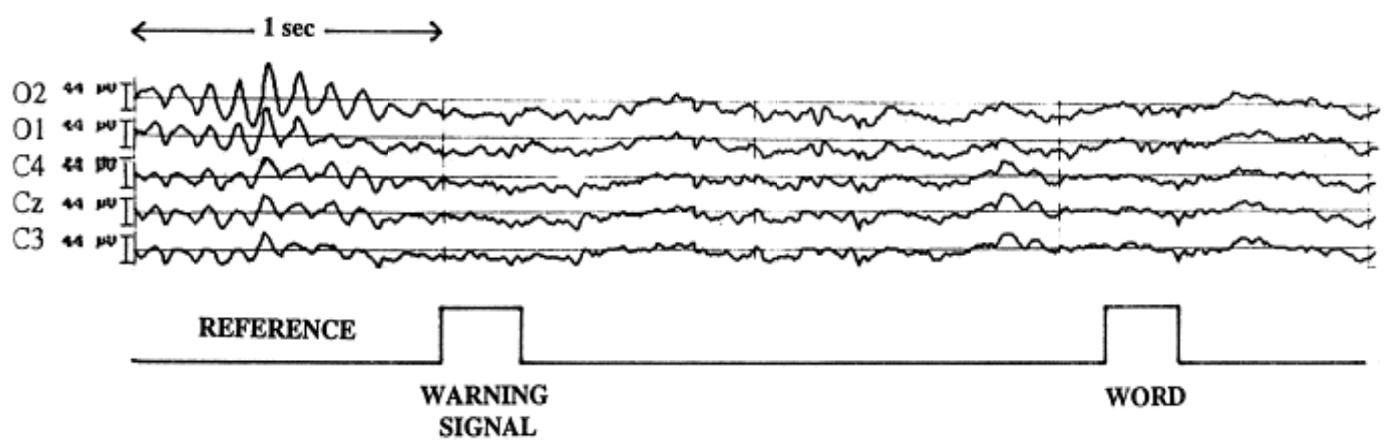

Figure 2. Typical example of an EEG epoch, showing the basic principle of alpha desynchronization. 

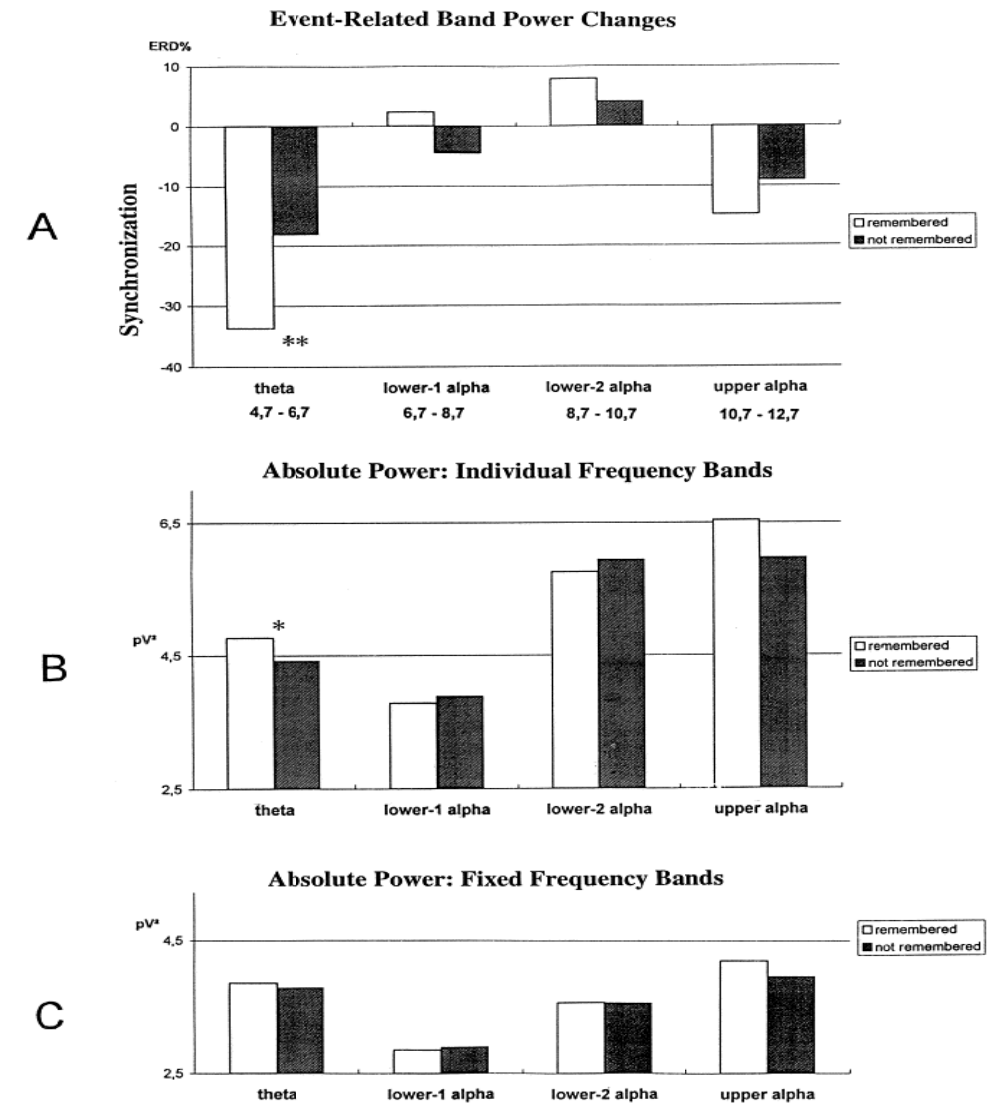

Figure 3. ERD during the encoding phase of an incidental memory task.

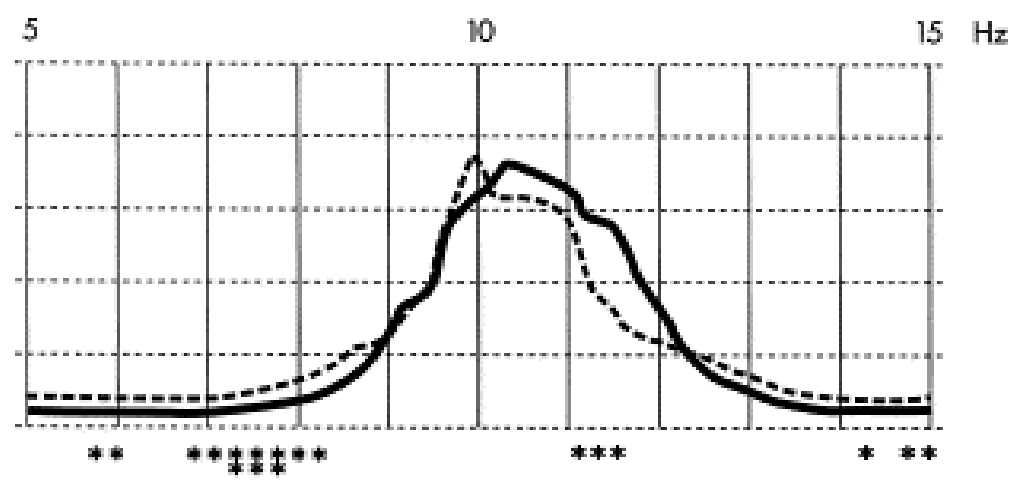

Figure 4. Normalized percent power for good and bad memory performers during memorizing words at $\mathrm{O} 1$. 